\title{
Feeling It Out: The Use of Haptic Visualization for Exploratory Geographic Analysis
}

\author{
Amy L. Griffin \\ Department of Geography \\ The Pennsylvania State \\ University \\ 302 Walker Building \\ University Park, PA 16802 \\ alg207@psu.edu
}

INTRODUCTION

"Haptic sensations are those sensations that are related to or based upon the sense of touch."
Visualization is often defined as the act or process of making something visible. MacEachren and Ganter (1990) have argued for an expanded definition of cartographic visualization that emphasizes the role of the map-reader's cognitive processes and schemata when creating visual representations. Cartographic visualization in this sense requires both the designer and the user to structure information and identify salient patterns. Processes of pattern identification and structuring are what help to provide insight in exploratory analysis. Pattern identification and information structuring need not, however, be limited to the visual realm. The use of haptic (both tactual and kinesthetic) information for representing geographic phenomena has been given limited attention as a method for exploring data, due to the difficulty of implementing such methods. However, advances in virtual reality technologies may soon make it possible to implement these variables in a system that creates exploratory geographic virtual environments. This paper explores those haptic variables that might be used to create such representations, and develops a haptic variable syntax for the representation of geographic information based on a logical analysis of the physiological properties of haptic sensation.

Keywords: haptics, cartographic representation, exploratory visualization, virtual reality

$\mathrm{V}$ isualization is often thought of as the act or process of making something visible. It is a technique used in many disciplines (including geography) to represent objects or concepts that do not necessarily exist in some tangible material form (e.g. fear spaces) or objects whose extent is so large that the human eye cannot inspect them from one vantage point (e.g. the continent of North America). Some scientists have argued that visualization is much more than the creation of visual artifacts; it is also "an act of cognition, a human ability to develop mental representations that allow us to identify patterns and create or impose order." (MacEachren and Ganter, 1990:66)

Visualization research, as the label implies, has focused on visual representations as a prompt to thinking. In the real world, however, we receive information through multiple sensory channels and can mentally represent our experiences in ways that take advantage of that multisensory experience. This paper focuses on the potential of haptic representation as a complement to visual representation.

Haptic sensations are those sensations that are related to or based upon the sense of touch. There are two primary types of haptic sensation: tactile and kinesthetic. Tactile sensations are those sensations that are perceived when the skin comes into contact with an object. Kinesthetic sensations are those that are mediated by receptors located in muscles and joints, and that are stimulated by bodily movements and tensions. That is, they are based on the forces of resistance we feel as a result of gravity or when we come into contact with an object. Because any postural position (even laying down) requires the compression of some muscles and joints, we 
constantly perceive kinesthetic information. In contrast, tactile sensations are perceived only when the skin comes into contact with another object.

This paper specifically explores the potential for using haptic sensations to represent and explore geographic phenomena, with particular emphasis on the potential that virtual reality technology offers for implementing haptic sensations. Haptic representations are considered because haptic perception is active and exploratory, making it a particularly intuitive medium for exploring data sets. Haptic representation allows a literal and direct interaction with the data. To support the use of haptic sensation, a haptic variable syntax for creating haptic representations of geographic data is proposed. This syntax was developed by systematic analysis of the physiological properties of haptic perception.

In addition to the potential that virtual environments offer for implementing haptic sensations, they are offered as a medium within which to create haptic representations because they allow the simultaneous use of multiple sensory modalities. This multisensory characteristic of virtual environments may enable users to take advantage of perceptual skills developed in their normal interactions in space and with objects.

The remainder of the paper will review the methods that cartographers have commonly used for representing geospatial information. Then a haptic variable syntax for creating haptic representations is proposed, along with potential applications of haptic sensations for representing geospatial information. Finally, issues associated with using haptic variables to represent geospatial information are discussed.

\section{Cartographic Use of Vision}

If the cognitive processes by which we make sense of spatial information are similar for different sense modalities, what cartographers have learned about using visual stimuli to represent spatial phenomena can inform our attempts to represent data using other sensory modalities. Several researchers have argued that these processes are similar for vision and touch. A useful starting point is a discussion of Bertin's (1983) visual variable syntax, and the expansion of this syntax by other researchers.

In his cartographic sign-system of visual variables, Bertin (1983) first identified the visual variables available to the information visualization designer (geographic position in the plane, size, value, texture, color, orientation and shape), and then articulated rules for the appropriate use of these variables, based on whether the data to be visualized are nominal, ordinal or quantitative. These rules were based on his logical analysis of the perceptual properties of potential variables rather than on an empirical analysis. ${ }^{1}$ In this analysis, Bertin classified visual variables based on whether the symbols are perceived as similar to, or different from each other; as ordered; or as proportional (i.e. the variable's perceptual character). His recommendation was that the perceptual character of the variable should be matched to the character of the data.

The dogmatic manner in which Bertin (1983) presented his proposed rule system led several cartographers to criticize and expand upon his system. Drawing upon Bertin's syntax [and on other work by Morrison (1974) and Caivano (1990)], MacEachren (1995) created an expanded visual variable syntax. His visual variables are location, size, crispness, resolution, transparency, color value, color hue, color saturation, texture, orientation, arrangement and shape. Some of the new variables in MacEachren's syntax have been added because of the impact that computer technologies have had on cartography (e.g. saturation; computer graphics programs now allow for separate control of all three color components-hue, value and saturation). Others have been added by dissolving one of Bertin's
"Haptic representations are considered because haptic perception is active and exploratory, making it a particularly intuitive medium for exploring data sets."

\section{CARTOGRAPHIC USE OF VISUAL AND NON-VISUAL SENSATIONS}

\author{
"... what cartographers have \\ learned about using visual \\ stimuli to represent spatial \\ phenomena can inform our \\ attempts to represent data using \\ other sensory modalities."
}


"Developing cartographic variable syntactics is important because they can increase the potential for consistent interpretation of cartographic representations."

\section{"Theories about the logical} interrelationships of map symbols and their referents are less well developed for other non-visual symbols than for visual symbols."

\section{"Geographers have only rarely considered using non-visual sensations to represent data for normal-vision users."}

variables (e.g. texture, which Caivano (1990) split into directionality, size and density). Still others have been developed as part of an effort to study the representation of uncertainty in data (e.g. crispness, resolution and transparency) (MacEachren, 1992). Other changes have involved identifying the three usability levels for each variable; usable/possible/impossible, rather than Bertin's two levels of acceptable/unacceptable.

The work of cartographers who have challenged or expanded Bertin's (1983) visual variable syntax has led to a richer understanding of how to match the map designer's objectives with what the map-reader is capable of perceiving. Developing cartographic variable syntactics is important because they can increase the potential for consistent interpretation of cartographic representations. If the relationships between the map symbols and their referents recognized by the cartographer differ from those recognized by the map reader (or different map readers recognize different relationships), the result will be a different (possibly incorrect) interpretation of the map symbols (MacEachren, 1995).

Theories about the logical interrelationships of map symbols and their referents are less well developed for other non-visual symbols than for visual symbols. The following two subsections focus on reviewing what has been hypothesized about non-visual representations of data and how they should be matched to their referents.

\section{Cartographic Use of Sound}

Geographers have only rarely considered using non-visual sensations to represent data for normal-vision users. One notable exception to this is Krygier's (1994) work on sound and geographic visualization. Krygier developed a set of sound variables by considering the different elements and characteristics of sound (location, loudness, pitch, register, timbre, duration, rate of change, order and attack/decay) that could be used to represent data and he suggested potential applications for which sound might be particularly useful.

A few geographers have created applications that represent geographic data with sonic variables. Wheless et al. (1996) integrated sound into their virtual reality visualization of the Chesapeake Bay ecosystem. They used sound to represent numerically generated salinity data (a change in pitch represented a change in salinity value). By "walking-through" the study area, researchers could hear changes in salinity while seeing the bay's topography, which helped them to interpret links between processes that occur in the bay's physical and biological systems. Fisher (1994) used both a single sound component (pitch) and multiple sound components (pitch, silence and duration) together to represent the classification uncertainty associated with each pixel in a remotely sensed image. The combination variable was designed to use multiple cues to emphasize the classification uncertainty. Although Fisher did not empirically test or experimentally compare his system to other potential systems (e.g. ones using an additional visual variable to represent the classification uncertainty), based on anecdotal evidence, he concluded that users found the system helpful.

Researchers in other disciplines have evaluated the effectiveness of using sound to represent data for several data exploration tasks. Pereverzev et al. (1997) found that by listening to an amplified microphone signal, they could identify predicted oscillations in super fluid helium that they could not detect visually. Flowers and Hauer (1995) examined the effectiveness of visual and auditory trend plots at conveying certain data characteristics (function slope, shape and magnitude). They found no significant differences between the ability of subjects to extract information from either the sonic or the visual version of the graph. In a later 
study, Flowers et al. (1997) evaluated their subjects' ability to estimate direction and magnitude of correlation between two data variables from visual and sonic scatter-plots. They found that there were no significant differences between modalities in estimating the magnitude of correlations. Cronly-Dillon et al. (1999) developed a system that enabled users to identify the salient shape features (i.e. outlines or edges) of visual images from sonic representations through the deconstruction of one or more sets of features into a sound pattern, and subsequent analysis of the sound patterns individually or in concert. Experimental testing of the system showed that allowing subjects to somehow segment the overall sonic representation facilitated the task of accurately analyzing and identifying the visual target.

As a group, the data sonification studies provide evidence that, for unpracticed users, sound can (at least in some instances) represent data as effectively as vision. The main implication of the study by Cronly-Dillon et al. (1999) is that there may be particular methods that map or graph readers can use to analyze images using sound (and perhaps other senses as well) accurately and efficiently; once such methods are identified, users can be trained to employ them. Finally, other studies (Fisher 1994; Wheless et al. 1996; Pereverzev et al. 1997) suggest that there may be instances (e.g. when visual representation channels are overloaded) in which sound is more effective than vision; however, this assertion has not been systematically tested.

\section{Cartographic Use of Haptics}

The success of using sound to represent data raises the question of whether other senses might also be used effectively. Although cartographers have given some attention to the use of haptic sensations for representing geographic data (in the form of Braille maps and other maps for the visually impaired (Weidel, 1983)), their use and potential for normal-vision map readers has not been comprehensively addressed.

The one exception to this is the set of elementary tactile variables that Vasconcellos (1991) developed by translating Bertin's visual variables into a tactile form. However, Vasconcellos did not identify any tactile variables that do not have a direct visual analog (other than, perhaps, elevation) ${ }^{2}$. That is, her variables included only those variables that are perceptible by both vision and touch, such as symbol size, value (e.g. vertical lines portrayed at increasing densities), texture (different textures), shape, and the orientation of symbols. By simply translating Bertin's visual variables, Vasconcellos failed to include any tactile variables that can only be perceived haptically (e.g. temperature, pressure or kinesthetic variables such as resistance or kinesthetic location). She also did not include recommendations for use of those variables she proposed, perhaps assuming that the cartographer would infer them from Bertin's recommendations.

The following haptic variables address these deficiencies because they correspond to the physiological properties of touch and kinesthetics as well as suggesting recommendations for their use. Little if any cartographic research has addressed the potential for using kinesthetic sensations to represent spatial data, nor the utility of tactile sensations in maps for normal-vision map readers.

\section{Identifying and Describing Haptic Sensations}

The first step in developing a haptic variable syntax requires identifying potential variables. Haptic perception is based on a combination of cutaneous (skin-derived) and kinesthetic sensations. Skin, the human body's largest sensory organ, is the organ associated with perceptual qualities
". . . there may be instances in which sound is more effective than vision; however, this assertion has not been systematically tested."
REPRESENTATION OF GEOGRAPHIC PHENOMENA USING HAPTIC SENSATIONS 
"There are three basic classes of cutaneous receptors: mechanoreceptors, thermoreceptors and nociceptors."

"A key step in designing any type of spatial data representation is deciding what types of symbolization are most appropriate for the data that are to be represented." such as touch, temperature, and pain. The average adult human's skin has a surface area of approximately $1.8 \mathrm{~m}^{2}$. Its several layers contain the sensory receptors responsible for the perception of touch, temperature and pain. These receptors are the structures that are responsible for translating mechanical, thermal, chemical and electrical energy into neural signals that are processed by the somatosensory area of the parietal lobe of the brain's cortex to produce the sensations of touch (Cholewiak and Collins, 1991: 26).

There are three basic classes of cutaneous receptors: mechanoreceptors, thermoreceptors and nociceptors. Mechanoreceptors respond to skin indentations (pressure), thermoreceptors to temperature change, and nociceptors to several types of intense stimulation such as high pressure, heat and/or burning chemicals (stimuli that may damage the skin). Because it is unlikely that a map reader would want to use painful sensations to represent data, further discussion of receptors is limited to mechanoreceptors and thermoreceptors.

Mechanoreceptors are responsible for four types of haptic sensations: vibration, flutter, buzz and pressure. Bolanowski et al. (1988) proposed a four-channel model of cutaneous mechanoreception based on correlations between physiological data and responses from psychophysically defined sensations. He proposed that each of these four sensations arises when a particular type of mechanoreceptor is stimulated.

Thermoreceptors allow us to perceive increasing and decreasing temperature, and can record (perceive) both the overall magnitude of warming or cooling, regardless of the exact spatiotemporal distribution of the thermal stimuli over the skin, as well as localizing high-level stimulation. Thus, skin can be described as having good spatial summation and poor localization capabilities at low levels of thermal intensity and poor spatial summation and good localization capabilities at high levels of thermal intensity. In contrast, vision has good spatial summation and good localization at intermediate ranges of stimulus intensity (e.g. normal daylight conditions), but localization abilities decrease as the stimulus intensity reaches either extreme (e.g. almost total darkness or very bright conditions).

Kinesthetic information is information that is generated about the position, posture and movement of various parts of our bodies as a result of our bodies' interaction with gravity. Because of Earth's gravitational pull, all bodily movements and postures involve tension, compression and twisting forces on our joints, muscles and limbs. The two basic types of kinesthetic sensations are joint position and joint movement. Now that types of haptic sensations have been identified and described, the following subsection proposes a haptic variable syntax.

\section{Haptic Variable Syntax}

A key step in designing any type of spatial data representation is deciding what types of symbolization are most appropriate for the data that are to be represented. Like vision or audition, the haptic sensations that might be incorporated into a spatial data representation can be decomposed, or separated into a number of different variables. They can be grouped into three primary categories: those derived from touch, those derived from visual analogs, and those derived from kinesthesia.

Several variables can be derived from the physiological possibilities of touch (as described in preceding section), such as vibration, flutter, pressure and temperature. Although it could be difficult to strictly separate out one touch variable from others (e.g. if the user touches a surface that is vibrating at a certain frequency, this surface will also have a perceivable tem- 
perature), it is possible to maximize the user's attention to one sensation as opposed to another by holding all but one constant. In the example of vibration and temperature, all the vibrating surfaces could be kept at the same temperature, preferably that of the user's skin; the user would then be more likely to attend to the vibratory stimulus.

Other variables can be derived from cartographic visual variables, such as location, size, texture/grain, shape (or form), orientation and elevation. What differentiates these variables from those based on the physiological properties of touch is that these variables can be perceived by both vision and touch. Although there have certainly been instances in which some tactile variables, such as temperature, have been visualized for analytical purposes, this usually involves a transformation of a thermal stimulus into a stimulus based on hue or value (a variable we can perceive visually). Some might also argue that it is possible to visually perceive temperature, but it could be argued that this happens only in special cases, such as in the glowing red of iron in a blacksmith's shop. There are many items, such as a plastic container that has been in the microwave for five minutes, whose appearance does not give us any information about its temperature-it could be hot or cool, depending on its material composition and the level of power the microwave was running at.

A third set of variables is composed of those that do not follow directly from either physiological possibilities or cartographic design. What separates these variables from the other two groups is that a movement of, or a change in the user's position, or in the position of a stimulus relative to the user is required in order for the variables to represent information. Friction is felt when the hand is moved across or through a surface, resistance can be felt when attempting to deform a surface and changes in kinesthetic location (location of the hand in relation to the body) can be used to compare data values to one another.

Figure 1 presents a haptic variable syntax that rates the appropriateness of each variable for each data type. This rating is based on a consideration of whether the variable's perceptual characteristics are ordered or differential (i.e. whether differences in the variable's symbolization are quantitative or qualitative). No distinction has been made between ordinal and numerical data because there is little empirical research that articulates a map reader's ability to make accurate quantitative estimations from haptic symbols without a legend referent.

The basic logic behind recommending that certain variables are appropriate or inappropriate for either nominal or ordinal variables is that if a variable that is perceived as ordered is used to represent nominal differences, the map reader may assume that there is also an implied order to the data when in fact, there is not. If the cartographer and the map reader use different map schemata when creating and interpreting maps, the information that the cartographer intended to present and what the map reader interprets from the map symbols may be quite different.

The "possible" category is reserved for those variables that may be able to effectively represent both types of data. For example, texture could represent ordinal data if the same texture pattern was used at different densities (e.g. diagonal hatch-marks like those in Figure 1, depicted with a different number of lines per inch for each ordinal category). However, texture could also be nominal if different pattern arrangements were used to represent different categories (e.g. diagonal hatch-marks and a checked pattern).

These recommendations are a hypothesis, and (like most similar syntactics) need to be empirically tested to verify their validity. One particular point of uncertainty is whether texture/grain and orientation are really
"A third set of variables is composed of those that do not follow directly from either physiological possibilities or cartographic design."

"If the cartographer and the map reader use different map schemata when creating and interpreting maps, the information that the cartographer intended to present and what the map reader interprets from the map symbols may be quite different." 


\section{Tactile Variables}

Nominal Ordinal

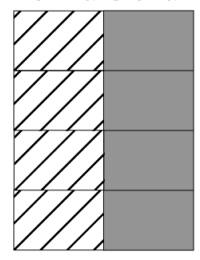

Vibration

Flutter

Pressure

Temperature

\section{Visual Analog Derived Variables}

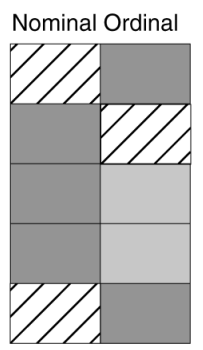

Size

Shape

Texture / Grain

Orientation

Elevation

\section{Kinesthetic Variables}

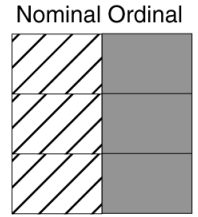

Resistance

Friction

Kinesthetic Location
"Haptic sensations may be best integrated into spatial data representations by implementing them in virtual reality environments."

Figure 1. Haptic variable syntax

appropriate for representing both nominal and ordinal data. Future empirical work that monitors and compares the types of errors and mistakes that map readers make when interpreting texture/grain and orientation as nominal or ordinal data may shed some light on this issue. Additional information can be gained in these experiments if map readers are asked to describe both their interpretations, and how they made those interpretations.

\section{Potential Applications of Haptic Variables}

Haptic sensations may be best integrated into spatial data representations by implementing them in virtual reality environments. Virtual environments are proposed as a medium within which to create haptic representations because they can simulate real environments; they can be highly interactive; and they allow the simultaneous use of multiple sensory modalities.

Given the potential for virtual environments to simulate real environments while simultaneously allowing users to experience these environments in ways that were previously not possible, the most useful appli- 
cation of haptic sensations in geographic visualizations may be iconic, particularly where it is possible to create an iconic haptic representation but not an iconic visual representation (e.g. resistance is used to represent the density of an air mass at a given location); in iconic representations of phenomena users can draw on their everyday experiences and interactions with the subject matter when making connections between events and/or states of the virtual environment. This may be especially applicable when users are trying to interpret complex outputs of numerical models. For example, the vibration variable might be used to visualize the output of a seismic model that predicts the intensity of shaking due to an earthquake; such visualization might be used to identify unexpected areas of strong shaking. Flutter might be used to represent the output of a model that predicts precipitation over time. Pressure could be used to visualize jet stream wind velocity and location. Imagine an animation of wind over time, in which the user could track the movement of the jet stream while looking at changes in other climatic variables. Temperature might be used to represent water temperature in a river, so that the user could track cold currents through space and time. Resistance could be used to represent measures of pollution; the more polluted an area, the more difficult it might be to walk through an area or move your hand through the area. Friction could be used to visualize terrain roughness, with an increase in friction corresponding to increasingly difficult terrain. The examples mentioned here are just a sample of the ways in which we might expect haptics to be implemented in the (relatively near) future. Current applications are constrained by the available haptic interface technology.

Most current virtual reality systems have limited capabilities for representing the haptic information that we perceive in our everyday experiences. Force-feedback and tactile display devices are often crude, and temperature simulation is not often integrated into force-feedback or tactile devices. ${ }^{3}$ Force-feedback devices are often powered by machines that generate and exert relatively small forces on a user when she puts pressure on an object or a surface. Because these forces are then smaller than what the object could withstand in real life, this often allows the user to inappropriately push her finger through a virtual object or surface that would normally be impenetrable to the force a finger can exert. Because there is a high density of mechanoreceptors in the human hand, the resolution of tactile display devices must be quite high in order for the sensations that describe a virtual object to feel the same as they would if the user was touching the real object with his or her bare hand. Improvements in small-scale electronics will allow the construction of denser arrays of tactile stimulators, and improved physical models of object shape and texture will increase the realistic character of haptic representations. Although current haptic representation capabilities do not provide a highly realistic experience, haptic interface technologies are still in the early stages of development and should continue to improve rapidly.

Haptic interfaces used in VR systems range from devices such as data gloves and data body-suits to computer mice and joysticks that reflect forces back on the user. Force-feedback joysticks or computer mice can be used to portray kinesthetic information, such as resistance and friction, with some also able to portray texture (Minsky and Ouh-young, 1990; Shi and Pai, 1997). Data gloves use fiber optic sensors to detect hand position, and rigid link exoskeletons provide force-feedback (Luecke and Chai 1997).

Tactile display systems can be used for both the hand and other parts of the body. They fall into three categories: shape-changing displays that control the deformation of the skin by applying forces (Monkman, 1992),

\section{"Current applications are constrained by the available haptic interface technology."}

\author{
"Tactile display systems can be \\ used for both the hand and other \\ parts of the body."
}


ISSUES IN USING HAPTICS TO REPRESENT GEOSPATIAL DATA

\author{
"An important issue for \\ designers who wish to create \\ haptic representations is what \\ humans can perceive \\ haptically."
}

\footnotetext{
"Without observing a passage of time, observations of change could not exist. Without space, there could not be any relationships between objects or surfaces, nor movement between locations."
}

vibrotactile displays that use an array of vibrating pins placed against the skin (Shimojo et al., 1997), and electrotactile displays that use surface electrodes to stimulate the skin (Tan et al., 1999).

Because one of the advantages of virtual environments is the literal way in which they allow users to interact with their data, those devices (e.g. joysticks, exoskeletons) which both accept the input of information and display attribute information are of the most interest for haptic representations of geospatial information.

This section describes a number of practical issues that need further consideration before haptic representation systems are implemented: (1) what humans can perceive haptically; (2) how to train map readers to read haptic representations of geospatial data; (3) potential interactions of vision and touch; and (4) the problem of cognitive sensory overload.

\section{Limits of Haptic Perception}

An important issue for designers who wish to create haptic representations is what humans can perceive haptically. This is a function of the number of receptors available for stimulation as well as of the number of stimulations a receptor can process as individual stimuli within a given period of time. Von Uexküll (1957) suggested a theory of perception based on functional cycles. This theory holds that the stimuli an organism can perceive are based on the organism's needs when interacting with the environment, and that for every perceptual cue the organism receives, there is an effector cue, which prescribes a course of action for the organism. Thus, in Von Uexküll's view, the functional tasks an organism can carry out are decided by the number and arrangement of their receptor cells.

In humans, haptic receptors are not evenly distributed throughout the body. For example, tactual acuity, which can be taken as a measure of the density of receptors in a given part of the body, is much higher for the tip of the index figure than for the back. This makes intuitive sense when you consider that our hands perform more complicated tasks than our backs, and therefore need to receive more precise perceptual cues about their interactions with the environment.

Both time and space are fundamental dimensions of our existence. Without observing a passage of time, observations of change could not exist. Without space, there could not be any relationships between objects or surfaces, nor movement between locations. Based on studies of several organisms, Von Uexküll also proposed that for each organism, time (as the organism experiences it) is divided into a series of moments whose length varies among organisms. The length of a moment is defined as the unit of time in which an indivisible elementary sensation can be perceived. For humans, he notes that one moment is $1 / 18$ of a second (55 milliseconds). That is, if two stimuli reach our receptors less than $1 / 18$ of a second apart, they will be perceived as a single sensation. He provided evidence that the length of this moment (1/18 of a second) holds for vision, sound and touch (Von Uexküll 1957). Other studies, however, have suggested that the length of this moment varies between modalities (i.e. its length depends upon which sensory modality is employed) (White 1963; Eriksen and Collins 1968) as well as on the combination of sensory modalities that are employed in the perceptual task (Dufft and Ulrich 1999). Although the different sensory modalities have different abilities to provide temporal, spatial and intensity information from perceptual stimuli, the input from these various sensory modalities are easily integrated (see more discussion of this below in Section 3.3, Interactions of Vision and Touch). 
Map Reader Ability to Read Haptic Maps

When research identifies more clearly what haptic representations uses can comprehend, then the efficacy of using such representations to construct knowledge from haptic spatial data representations becomes a central question. Most cartographic research on haptic sensations has focused on their potential utility in maps and graphics for the visually impaired and the blind. This work has addressed two topics: the extent to which tactile maps support spatial cognition in the visually impaired and the psychophysical aspects of reading tactile maps. Much of this literature has examined the use of tactile mobility maps for reference and navigation, rather than thematic maps. Because haptic cartographic research has focused on these issues, less is known about map readers' ability to identify patterns or trends (and thereby construct knowledge) from data represented with haptic symbols than is known about map readers' ability to retrieve a particular piece of information from the map or use the map to solve a particular navigation problem.

Several authors have examined the ability of blind or visually impaired subjects to retrieve and synthesize spatial information from tactile maps. When using the sense of touch, the area on a tactile map that can be studied (i.e. that can yield tactile perceptual information) at one moment in time is limited to the size of the surface area of the skin that is applied to the map. Because of this limitation, many stimuli in tactile maps must be perceived sequentially. ${ }^{4}$ The tactile map reader must then integrate them into a coherent whole in memory, just as sighted persons must when navigating a large space (containing barriers that prevent seeing it all at once) without the help of a map. Such a synthesis of spatial information may be most easily accomplished when some knowledge of the space's overall structure is available prior to examining the tactile map (Andrews 1983). In sighted persons, viewing a map provides this overall structure; for most visual maps, it is understood that space on the map represents space in the environment (Dodds 1988).

Castner (1983) suggested, and Unger et al. (1997) demonstrated empirically, that tactile map-learning strategies involving active exploration of maps (e.g. those that relate map elements in space, identify patterns and establish an overall structure) are more successful at promoting knowledge of the overall structure of a space than sequential (route-based) explorations of maps. Unger et al. postulated that this difference exists because the sequential exploration of a map is more likely to prompt the map reader to consider local spatial relationships (i.e. between a feature and adjacent features that also lie on the route) than global spatial relationships (i.e. relationships between non-adjacent features and/or features that do not lie along the route). The implication of Unger et al's findings is that if the map reader's goal is to obtain an overall knowledge of the space, active explorations of spaces should be promoted for haptic visualization users. In normal-vision users, however, this may be less important if they can visually extract knowledge of the space's overall structure.

If map readers cannot differentiate among the symbols used in a map, the map will not be effective. Psychophysical studies of maps attempt to discover how readers interpret different stimuli presented on the map. The results of the few psychophysical studies directed to evaluating the efficacy and appropriateness of particular haptic symbols for representing different types of geographic data provide some useful guidance for creating haptic thematic maps. Thompson (1983) carried out a study to determine whether range-graded graduated point symbols could be used effectively in maps designed for the blind. He found that this symbolization was effective only if a full legend (i.e. a legend that included exem-
"Most cartographic research on haptic sensations has focused on their potential utility in maps and graphics for the visually impaired and the blind."

". . . if the map reader's goal is to obtain an overall knowledge of the space, active explorations of spaces should be promoted for haptic visualization users."

"If map readers cannot differentiate among the symbols used in a map, the map will not be effective." 
"Knowledge of a space's overall structure does not necessarily have to be obtained visually."

". . for sighted users, the main issue is whether a system that employs some combination of modalities has an advantage over a vision-only system." plars of all symbol sizes used in the map) was included. Andrews (1983) performed a similar study with similar results. The conclusion that can be drawn from their results is that map readers are not as good at estimating numerical data values from haptic symbols as they are at matching symbols with values included in the legend. The same conclusion has been reached regarding graduated visual symbols, suggesting that more legend anchors could improve magnitude estimations (Cox 1976). To my knowledge, there have not been any studies that have compared the ability of subjects to judge magnitudes from visual and tactile symbols.

Although cartographic research on the use of haptic sensations for visualizing data has traditionally been directed to visually impaired and blind map readers, the availability of virtual reality technologies is now producing environments within which haptic sensations may be useful in applications for normal vision users. Combining visual and haptic sensations in virtual reality systems may be especially fruitful, particularly in light of research by Unger et al. (1997). Virtual reality can provide such an environment, in which vision is used to acquire an understanding of the space's structure, and both vision and haptics are used to explore relationships within this space. Knowledge of a space's overall structure does not necessarily have to be obtained visually. A verbal description of the relative locations of objects within the space could also provide this knowledge. However, Blades et al. (1999) have shown that viewing a map of a space is more effective than touch at promoting survey knowledge of that space. Therefore, the implication is that vision should be used for this task in virtual environments.

Interactions of Vision and Touch

Although a purely haptic visualization system might be created for the visually impaired, for sighted users, the main issue is whether a system that employs some combination of modalities has an advantage over a visiononly system. If there is an advantage to using multiple modalities, the next question is how the modalities should be combined.

One particularly important intersensory relation is that between touch and vision. There are four potential relationships between these two modalities:

Vision and touch operate independently, with little or no interaction. Vision allows for more accurate perception than touch.

Touch allows for more accurate perception than vision.

Vision and touch interact in complex ways (e.g. interference, redundancy, complementarity).

Several researchers have concluded that vision and touch are equally capable of discriminating between textures accurately (Lederman and Abbott 1981; Jones and O'Neill 1985). In a later experiment, Lederman et al. (1986) showed that vision and touch process texture in different ways, which depend on the task at hand. For example, they found that texture is used visually to divide spatial arrays of surfaces into chunks, while touch is used to examine surface properties (e.g. is the surface sharp or smooth?). Heller (1982) found that a combination of the two modalities improved performance in processing texture, and concluded that this was due not to the added visual information related to the texture, but to the ability of vision to help guide the hand in its exploration of the texture.

Warren and Rossano (1991) summarized the literature on the interplay of vision and touch with respect to several other variables, such as tilt, size and length and shape. They found that tactile judgment of tilt, size 
and length is as accurate as visual judgment, but that tactile judgment of shape is less accurate than visual judgment (for shape). They also found that when subjects were given conflicting information, vision tended to dominate the judgment.

Several authors have noted that the different sensory modalities are more appropriate for different tasks (e.g. touch - texture, vision - spatial location, and audition - temporal rate) because each sense is differently precise for perception of different events (Freides 1974; Welch and Warren 1980); this is presumably because each human sensory system has evolved for different purposes, but may also be reinforced by repeated use of one modality for a particular type of task. Welch and Warren (1980) suggested that this differential precision encourages us to attend more to those senses that are most precise for the task at hand. Because of this specialization, it is necessary to take into account the purpose of the visualization task and its information requirements (e.g. is the information distributed temporally, spatially or spatiotemporally?) when designing visualization systems.

There are three general ways haptics could be implemented in a virtual environment: the representation could consist solely of one data variable represented haptically (only one variable is represented; no other modalities are employed); the representation could consist of a combination of data set variables in which one variable is represented haptically and others are represented using auditory or visual cues; or it could use haptics as a supplement to visual information if one data variable is represented using multiple modalities. Although the first possibility may have important applications for the visually impaired, most people with normal-vision have little formal training in interpreting data that are represented only through haptic sensations. For this reason, it may be most fruitful to create virtual environments in which several data attributes are represented using different modalities, which allows users to concurrently observe the distribution of values of the haptically represented attribute within the context of the values of one or more visually represented attributes, or to represent one attribute redundantly. Cartographers have already shown that, at least for one visual variable combination (size and value), such redundancy (if created through multiple visual cues) significantly improves the accuracy and response time of interpretations made by map readers (Dobson 1983). Although to my knowledge no cartographers have empirically investigated the interactions of multimodal variable integration, some psychological experiments have shown that combinations of visual and auditory stimuli improve task response times (Dufft and Ulrich 1999).

Haptic information is most often implemented in virtual environments as a supplement to visual images. Including haptic information creates a more realistic, more completely immersive user-experience. While the use of haptic information to create an experience that more closely matches reality is itself useful, virtual reality technology's most important contribution to scientific and geographic visualization may lie in its ability to represent abstract data with haptics or other sense modes. This could be accomplished by visualizing data that we cannot see in the course of our everyday lives (e.g. air pressure). Because air pressure differences do not normally occur rapidly enough for us to notice them (while standing in one location), and because it is unlikely that we would be able to remember the sensation that a given air pressure produced long enough to travel to a location where the air pressure would feel different, we typically visualize air pressure differences using vision. However, in a virtual environment, both space and time can be compressed, and the magnitude
". . . most people with normal-vision have little formal training in interpreting data that are represented only through haptic sensations."

"Haptic information is most often implemented in virtual environments as a supplement to visual images." 
"A map that displays more than two or three attributes with some combination of different visual variables quickly becomes much more difficult for the map reader to interpret."

“. . more perceptual cues do not necessarily allow the user to process more information. The amount of information that the user can effectively process is likely to be task-specific ..." of air pressure differences can be exaggerated, so that when moving from one location to another, there could be a perceptible change in the amount of pressure we feel on our bodies (that corresponds to the change in air pressure in the data set).

Cognitive Sensory Overload and Cartographic Legends

A final important issue in designing haptic representations of geospatial data is that of cognitive sensory overload - a condition in which the map reader cannot process all of the information represented in the display, and in which adding more information to the display may actually lead to a decrease in the amount of information the map reader can effectively process. Cartographers are already familiar with this issue in the intramodal case (i.e. within one sense) for vision in a given time period. A map that displays more than two or three attributes with some combination of different visual variables quickly becomes much more difficult for the map reader to interpret. Certainly, we could expect that this difficulty would also extend to haptic variables, and may be even more severe for that case. The difficulties associated with interpreting a visual representation of multiple attributes are at least attenuated to some degree by the semi-permanent nature of the display-the map reader does not have to store as much information in her working memory, as the display itself can help to fulfill this function. A haptic display, on the other hand does not leave a semi-permanent trace to serve as a store of working memory, so we might expect that difficulties in interpreting displays that represent multiple attributes haptically to be even more severe than those for vision. As such, it seems appropriate to restrict the use of haptic variables to the display of one attribute at a time.

Cognitive sensory overload may also occur intermodally (i.e. between senses). A commonly reported scenario occurs when driving a high-tech car of the future. A study by the Oak Ridge National Laboratory examined how much of a driver's cognitive capacity went to dealing with a cell phone, a forward collision-warning system, a navigation system and an Internet-equipped computer screen while driving, and what portion of their mental capacity was devoted to the task at hand (i.e. driving the car) (ITS America 2001). The scientists found that drivers were better at attending to the multiple devices (often using multiple senses, such as vision and audition) when they could finish one task before dealing with another. The implication this study has for designing visualization systems is that more perceptual cues do not necessarily allow the user to process more information. The amount of information that the user can effectively process is likely to be task-specific (i.e. it may be easier for a user to find anomalies in a multisensory representation than to determine whether a correlation exists between two variables). Therefore, user control of the system is important for helping user's manage the cognitive load associated with integrating and processing data from multisensory perceptual cues.

We might also expect that in some cases, multisensory perceptual cues will enhance or augment cognition. For example, nurses in the emergency room often rely on changes in the sounds emitted from machines that monitor the patient's status (along with visual cues) to alert them to a patient's deteriorating condition. Without such sonic input, it is likely that a nurse would not be able to effectively monitor the condition of several patients at once. A cartographic scenario in which a similar process could potentially occur might be examined by using Fisher's (1994) vision and sound representation system is using sound to alert a user that a remotely 
sensed pixel's classification uncertainty has exceeded a user-specified threshold.

We may find that the way we design intermodal representations of geospatial data will have an important effect on whether they produce a condition of cognitive sensory overload in the user. One particular area of concern is in legend design. For example, when a map reader is trying to understand the visual stimuli she sees in a map, she can refer to the legend at any time without losing her place in the mapped distribution, or changing the perceptual stimulus she is attending to. However, with a haptic representation, once the map reader's hand leaves a particular location on the map display, the sensation she feels will also change. Thus, reading the map and referring the legend with the same hand may be very difficult. For this reason, the map reader may use one hand to explore the display and the other as the "legend hand" while exploring haptic representations. The legend could be set to display one reference value or the entire range of values represented in the display, depending on the user's preference and/or the task at hand. Once haptic displays begin to be implemented, it would be useful to design a variety of legends and test their effectiveness for several map reading tasks.

The potential for including touch as a channel for geographic visualization, either as a supplement to visual representation or on its own has not yet been widely explored. With advances in virtual reality technology and computing power, more sophisticated representations of data with haptics will be possible. It is important to think carefully about how the various properties of haptic perception can best be applied to representing geographic data, as well as to evaluate their effectiveness in representing geographic data once haptic representation capabilities are implemented in visualization systems. Empirical testing, both of the effectiveness of the proposed haptic variable syntax and of haptic variables themselves (individually and in combination with visual and/or other sense variables) would help to identify areas for their appropriate implementation and application.

Andrews, S. K. 1983. Spatial cognition through tactual maps. Proceedings of the First International Symposium on Maps and Graphics for the Visually Handicapped. 30-40. Washington, D.C.: Association of American Geographers.

Bertin, J. 1983. Semiology of Graphics: Diagrams, Networks, Maps. Madison, Wisconsin: University of Wisconsin Press. Translated by William Berg from Semiologie Graphique (1967). Paris: Editions Gauthier-Villars.

Blades, M., S. Ungar and C. Spencer. 1999. Map use by adults with visual impairment. Professional Geographer. 41(4): 539-53.

Bolanowski, S. J., Jr., G. A. Gescheider, R. T. Verillo and C. M. Checkosky. 1988. Four channels mediate the mechanical aspects of touch. Journal of the Acoustical Society of America. 84(5): 1680-94.

Brewer, C. A., A. M. MacEachren, L. W. Pickle and D. Hermann. 1997. Mapping mortality: evaluating color schemes for choropleth maps. Annals of the Association of American Geographers. 87(3): 411-38.
"With a haptic representation, once the map reader's hand leaves a particular location on the map display, the sensation she feels will also change. Thus, reading the map and referring the legend with the same hand may be very difficult."

SUMMARY

REFERENCES 
Caivano, J. L. 1990. Visual texture as a semiotic system. Semiotica. 80(3/4): $239-52$.

Castner, H. W. 1983. Tactual maps and graphics: some implications for our study of visual cartographic communication. Cartographica. 20(3): 1-16.

Chang, K.-T. 1977. Visual estimation of graduated circles. Canadian Cartographer. 14: 130-8.

Cholewiak, R. W. and A. A. Collins. 1991. Sensory and physiological bases of touch. In M. A. Heller and W. Schiff (eds.) The Psychology of Touch. Hillsdale: Lawrence Erlbaum Associates, Publishers.

Cox, C. W. 1976. Anchor effects and the estimation of graduated circles and squares. American Cartographer. 3: 65-74.

Cronly-Dillon, J., K. Persaud and R. P. F. Gregory. 1999. The perception of visual images encoded in musical form: a study in cross-modality information transfer. Proceedings of the Royal Society of London, Series B. 266: 2427-33.

Dobson, M. W. 1983. Visual information processing and cartographic communication: the utility of redundant stimulus dimensions. In D. R. F. Taylor (ed.) Graphic communication and design in contemporary cartography, Volume 2, Progress in contemporary cartography. New York, Wiley.

Dodds, A. 1988. Tactile maps and the blind user: perceptual, cognitive and behavioral factors. Proceedings of the Second International Symposium on Maps and Graphics for Visually Handicapped People. 30-6. London: University of Nottingham.

Dufft, C. C. and R. Ulrich. 1999. Intersensory facilitation: visual accessory signals can also shorten reaction time. Zeitschrift für experimentelle Psychologie. 46(1): 16-27.

Eriksen, C. W. and J. F. Collins. 1968. Sensory traces versus the psychological movement in the temporal organization of form. Journal of Experimental Psychology. 77: 376-82.

Fisher, P. 1994. Hearing the reliability in classified remotely sensed images. Cartography and GIS. 21(1): 31-6.

Flannery, J. 1971. The relative effectiveness of some common graduated point symbols in the presentation of quantitative data. Canadian Cartographer. 8: 96-109.

Flowers, J. H., D. C. Buhman and K. D. Turnage. 1997. Cross-modal equivalence of visual and auditory scatterplots for exploring bivariate data samples. Human Factors. 39(3): 341-51.

Flowers, J. H. and T. A. Hauer. 1995. Musical versus visual graphs: Crossmodal equivalence in perception of time series data. Human Factors. 37(3): 553-69.

Freides, D. 1974. Human information processing and sensory modality: Crossmodal functions, information complexity, memory and deficit. Psychological Bulletin. 81: 284-310. 
Heller, M. A. 1982. Visual and tactual texture perception: Intersensory cooperation. Perception \& Psychophysics. 31: 339-44.

ITS America. 2001. High-tech cars could bring cognitive overload. http:/ / www.itsa.org/ITSNEWS. NSF/a619bd3fc912d6f38525658d00073cd1/ 4d4a2ccf01557420852569dd00517abe?OpenDocument

Jones, B. and S. O'Neill. 1985. Combining vision and touch in texture perception. Perception and Psychophysics. 37: 66-72.

Kimerling, A. J. 1985. The comparison of equal-value gray scales. American Cartographer. 12: 132-42.

Krygier, J. 1994. Sound and geographic visualization. In A. M. MacEachren and D. R. F. Taylor (eds.) Visualization in Modern Cartography. Oxford, UK: Elsevier.

Lederman, S. J. and S. G. Abbott. 1981. Texture perception: studies of intersensory organization using a discrepancy paradigm and visual vs. tactual psychophysics. Journal of Experimental Psychology: Human Perception and Performance. 47: 54-64.

Lederman, S. J., G. Thorne and B. Jones. 1986. Perception of texture by vision and touch: multidimensionality and intersensory integration. Journal of Experimental Psychology: Human Perception and Performance. 12: 169-80.

Luecke, G. R. and Y.-H. Chai. 1997. Contact sensation in the synthetic environment using the ISU force reflecting exoskeleton. IEEE Annual Virtual Reality International Symposium. 192-8. Albuquerque, NM: IEEE Computer Society Press.

MacEachren, A. M. 1992. Visualizing uncertain information. Cartographic Perspectives. 13: 10-19.

MacEachren, A. M. 1995. How Maps Work. New York: Guilford Press.

MacEachren, A. M. and J. H. Ganter. 1990. A pattern identification approach to cartographic visualization. Cartographica. 27(2): 64-81.

Minsky, M., and M. Ouh-young. 1990. Feeling and seeing: Issues in force display. Computer Graphics. 24(2): 235-43.

Monkman, G. J. 1992. An electrorheological tactile display. Presence. 1(2): 219-28.

Morrison, J. L. 1974. A theoretical framework for cartographic generalization with the emphasis on the process of symbolization. International Yearbook of Cartography. 14: 115-27.

Ottensmeyer, M. P. and K. J. Salisbury. 1997. Hot and cold running VR: Adding thermal stimuli to the haptic experience. Proceedings of the Second PHANToM User's Workshop. 34-7. Dedham, MA: Endicott House. 
Pereverzev, S. V., A. Loshak, S. Backhaus, J. C. Davis, and R. E. Packard. 1997. Quantum oscillations between two weakly coupled reservoirs of superfluid He-3. Nature. 388: 449-451.

Shi, Y. and D. K. Pai. 1997. Haptic display of visual images. IEEE Annual Virtual Reality International Symposium. 188-91. Albuquerque, NM: IEEE Computer Society Press.

Shimojo, M., M. Shinohara and Y. Fukui. 1997. Shape identification performance and pin-matrix density in a 3-dimensional tactile display. IEEE Annual Virtual Reality International Symposium. 180-7. Albuquerque, NM: IEEE Computer Society Press.

Tan, H. Z., N. I. Durlach, C. M. Reed and W. M. Rabiowitz. 1999. Information transmission with a multifinger tactual display. Perception $\mathcal{E}$ Psychophysics. 61(6): 993-1008.

Thompson, N. R. 1983. Tactual perception of quantitative point symbols in thematic maps for the blind. Proceedings of the First International Symposium on Maps and Graphics for the Visually Handicapped. 103-13. Washington, D.C.: Association of American Geographers.

Ungar, S., M. Blades, and C. Spencer. 1997. Strategies for knowledge acquisition from cartographic maps by blind and visually impaired adults. The Cartographic Journal. 34(2): 93-110.

Vasconcellos, R. 1991. Knowing the Amazon through tactual graphics. Proceedings of the International Cartographic Association. 206-10. Bournemouth: International Cartographic Association.

Von Uexküll, J. 1957. A stroll through the worlds of animals and men: A picture book of invisible worlds. In C.H. Schiller (ed.) Instinctive Behavior. Madison, CT: International Universities Press.

Warren, D. H. and M. J. Rossano. 1991. Intermodality relations: Vision and touch. In M. A. Heller and W. Schiff (eds.) The Psychology of Touch. Hillsdale: Lawrence Erlbaum Associates, Publishers.

Weidel, J., Ed. 1983. Proceedings of the First International Symposium on Maps and Graphics for the Visually Handicapped. Washington, D.C.: Association of American Geographers.

Welch, R. B. and D. H. Warren. 1980. Immediate perceptual response to intersensory discrepancy. Psychological Bulletin. 88: 638-667.

Wheless, G. H., C. M. Lascara, A. Valle-Levinson, D. P. Brutzman, W. Sherman, W. L. Hibbard and B. E. Paul. 1996. Virtual Chesapeake Bay: Interacting with a coupled physical/biological model. IEEE Computer Graphics and Applications. 16(4): 52-7.

White, C. 1963. Temporal numerosity and the psychological unit of duration. Psychological monographs. 77: 1-37. 
${ }^{1}$ Empirical work testing some of Bertin's contentions has been undertaken (c.f. studies by Brewer et al. (1997), Flannery (1971), Chang (1977) and Kimerling (1985)).

${ }^{2}$ Elevation can certainly be perceived visually if the map reader has access to a $3 \mathrm{D}$ representation.

${ }^{3}$ One notable exception to this is the addition of thermal stimuli to the PHANToM haptic interface (Ottensmeyer and Salisbury 1997).

${ }^{4}$ Although visual stimuli in maps are also perceived sequentially, most visual scanning tasks (for most individuals) are probably performed much more quickly than tactile scanning processes because larger chunks of the scene are sensed at once, and vision has greater acuity. 